\title{
Recurrent Idiopathic Orbital Myositis: Two Case Reports
}

\author{
Pinar ERÖZ* and Mehmet Atila Argin \\ Department of Ophthalmology, Mersin University Faculty of Medicine, Mersin, Turkey
}

*Corresponding author: Pinar Eröz, Department of Ophthalmology, Mersin University Faculty of Medicine, Mersin, Turkey

\begin{abstract}
Idiopathic orbital myositis is a subtype of idiopathic orbital inflammatory diseases characterized by inflammation of the extraocular muscles. The diagnosis of the disease is made clinically and radiologically. Clinically characterized by periorbital edema, ptosis, propitosis, diplopia and painful eye movements. The diagnosis is made by observing the enlargement of the involved muscles in the orbital CT. The most common differential diagnosis is thyroid ophthalmopathy. In this study, we presented two patients with idiopathic orbital myositis who were referred to our clinic with complaints of eye rash, eye movement pain, bilateral visual acuity. Idiopathic orbital myositis was diagnosed and $1 \mathrm{mg} /$ $\mathrm{kg} / \mathrm{day}$ oral prednisolone was started. Symptoms of the patients regressed with oral corticosteroid therapy.
\end{abstract}

\section{Keywords}

Orbital myositis, Steroids

\section{Introduction}

Idiopathic orbital myositis is a sub-form of idiopathic orbital inflammatory diseases characterized by inflammation of one or more extraocular muscles without any cause. It was first described by Gleason in 1903 [1]. It most commonly affects individuals in the thirties. Female/male incidence is $2 / 1$. Clinically recurrent unilateral pain is ptosis, proptosis, periorbital edema, chemosis, diplopia and conjunctival hyperemia on the affected muscle [2,3]. It may occur as acute or chronic. Histopathologically, there are polymorphic lymphocyte infiltration with varying degrees of fibrosis [4]. The diagnosis of the disease is made clinically and radiologically. Treatment includes systemic steroids. In this study, two cases that presented to the clinic with redness in the eye and pain in eye movements were discussed.

\section{Case 1}

34-year-old woman presented with redness in her right eye, pain in her eye movements, and swelling in the eyelid. The patient presented to the external center with similar symptoms two years ago and his complaints regressed after oral corticosteroid treatment. On ophthalmologic examination, both eyes had 20/20 visual acuity. In the right eye, conjunctival hyperemia, abduction and adduction restriction, pain in the eye movements, periorbital edema and proptosis were present and posterior segment examination was normal. The right glob was $2 \mathrm{~mm}$ proptotic. His vitals were stable. Orbital CT showed irregular thickening of the right lateral rectus muscle.

Hemogram, sedimentation, CRP, ANA, ANCA, RF, antihiston antibody, anti dsDNA, anti-Scl70, antithromoglobulin, anti TPO, HLA B27, HLAB51, ferritin, folic acid, vit $B 12$, complete urine analysis were performed. The patient was consulted to the rheumatology department. In laboratory results, all values are within normal limits. Chest X-ray and lumbosacral graphy was normal.

Rheumatological pathology was not considered. Idiopathic orbital myositis was diagnosed and $1 \mathrm{mg} / \mathrm{kg} /$ day oral prednisolone was started. The patient was followed-up. Prednisolone was reduced $16 \mathrm{mg}$ per week. Clinical findings of the patient regressed and no recurrence was observed. The patient's controls are continuing in our clinic.

\section{Case 2}

49-year-old male patient was referred to our clinic from the external center with the complaint of pain in his right eye for 3 days, restriction of eye movements and ptosis. He had been treated with the same complaints 3 years ago with the oral corticosteroid treatment. On ophthalmologic examination, both eyes had $20 / 20$ visual acuity. right ptosis, chemosis, conjunctival 
hyperemia, and eyelid edema. The posterior segment examination was normal. In the right eye, he had superior visual impairment, pain in eye movements, periorbital edema, and proptosis. The right glob was proptotic. Bilateral direct and indirect light reflexes were positive. His vitals were stable.

Magnetic resonance imaging of the patient showed thickening of the superior rectus muscle. Biochemistry tests for the etiology of orbital inflammation were requested and the patient was consulted to the rheumatology department. All values were within normal limits in laboratory results. Chest X-ray and lumbosacral graphy were normal. $1 \mathrm{mg} / \mathrm{kg} /$ day oral prednisolone was initiated with the diagnosis of idiopathic orbital myositis. During follow-up, the patient's clinical findings regressed.

Idiopathic orbital myositis is a sub-form of idiopathic orbital inflammatory diseases. Primary extraocular muscles, the most common medial rectus, followed by superior rectus, inferior rectus and inferior oblique muscle. The most common differential diagnosis is thyroid ophthalmopathy. Imaging methods have an important role in the differential diagnosis of these two diseases. Although there is no rule, in the presence of thyroid ophthalmopathy there is a diffuse and regular involvement of the extraocular muscles, while the tendons of the muscles are not kept [5]. In orbital myositis, muscle involvement is more irregular and has tendon involvement. Laboratory findings may be normal in $10 \%$ of patients in thyroid ophthalmopathy [6]. The patient's thyroid hormone test values are normal and they cannot rule out the diagnosis of thyroid ophthalmopathy. In the radiological imaging, the tendon involvement and the irregular involvement of the muscles removed the diagnosis of thyroid ophthalmopathy. Orbital myositis has characteristic orbital and periorbital pain, restriction of ocular movements, increased pain with eye movements, diplopia, proptosis, eyelid swelling, conjunctival hyperemia and sometimes palpable mass. It is usually idiopathic. It may develop secondary to systemic diseases and infectious conditions. Systemic diseases may be associated with polymyositis, thyroid-dependent eye disease, psoriatic arthritis, rheumatoid arthritis, Kawasaki disease, Scleroderma, SLE, Crohn's disease, Ankylosing spondylitis. It may be associated with Lyme disease, Herpes Zoster ve Whipple disease. However, many inflammatory, vascular, neoplastic and infectious conditions affecting extraocular muscles and other orbital tissues may be confused with orbital myositis. The diagnosis is made by observing the enlargement of the involved muscles in the or- bital CT. Systemic steroids, NSAIDs, radiotherapy and surgery are included in the treatment. Possible autoimmune pathologies were excluded with clinical and laboratory findings. No intracranial pathology was found in the cranial MRI performed to exclude intracranial space occupying aneurysm, arteriovenous fistula and cavernous sinus thrombosis in the differential diagnosis of painful ophthalmoplegia. The dramatic response to corticosteroid is considered as a pathognomic finding for idiopathic orbital myositis [8]. Although fast and good response to corticosteroids in orbital myositis, it is recommended that steroid treatment should be continued for 4 to 6 weeks and stopping gradually because of the possibility of recurrence. Some groups of patients may experience steroid dependence or resistance. There are reports of useful intraorbital injection of low-dose radiotherapy, immunomodulatory agents and steroids in alternative therapy $[8,9]$. The patients were first given $1 \mathrm{mg} / \mathrm{kg} /$ day oral steroid, for 1 week and prednisolone was stopped by reducing $16 \mathrm{mg}$ per week. Systemic corticosteroid treatment was partially responded. In the follow-up, maintenance therapy was gradually reduced and stopped.

As a result, idiopathic orbital myositis should be kept in mind as a differential diagnosis in patients presenting with periorbital edema, ptosis, proptosis, diplopia, conjunctival hyperemia and edema and painful eye movements.

\section{References}

1. Gleason J (1903) Idiopathic myositis involving the extraocular muscles. Ophthalmic Rec 12: 471-478.

2. Moorman CM, Elston JS (1995) Acute orbital myositis. Eye (Lond) 9: 96-101.

3. Maurer I, Zierz S (1999) Recurrent orbital myositis: report of a familial incidence. Arch Neurol 56: 1407-1409.

4. Mahr MA, Salomao DR, Garrity JA (2004) Inflammatory orbital pseudotumor with extension beyond the orbit. Am J Ophthalmol 138: 396-400.

5. Rothfus WE, Curtin HD (1984) Extraocular muscle enlargement: a CT review. Radiology 151: 677-681.

6. Hertz S, Means JH, Williams RH (1941) Graves' disease with dissociation of thyrotoxicosis and ophthalmopathy. West J Surg 49: 493-498.

7. Rubin PA, Foster CS (2004) Etiology and management of idiopathic orbital inflammation. Am J Ophthalmol 138: 1041-1043.

8. Shah SS, Lowder CY, Schmitt MA, Wilke WS, Kosmorsky GS, et al. (1992) Low-dose methotrexate therapy for ocular inflammatory disease. Ophthalmology 99: 1419-1423.

9. Bullen C L, Young B R (1982) Chronic orbital myositis. Arch Ophthalmol 100: 1749-1751. 